\title{
NEW CLASSES OF GENERALIZED INVEX MONOTONICITY
}

\author{
B. XU AND D. L. ZHU
}

Received 26 December 2004; Accepted 16 August 2005

This paper introduces new classes of generalized invex monotone mappings and invex cocoercive mappings. Their differential property and role to analyze and solve variationallike inequality problem are presented.

Copyright (C) 2006 B. Xu and D. L. Zhu. This is an open access article distributed under the Creative Commons Attribution License, which permits unrestricted use, distribution, and reproduction in any medium, provided the original work is properly cited.

\section{Introduction}

Variational inequalities theory has been widely used in many fields, such as economics, physics, engineering, optimization and control, transportation $[1,4]$. Like convexity to mathematical programming problem (MP), monotonicity plays an important role in solving variational inequality (VI). To investigate the variational inequality, many kinds of monotone mappings have been introduced in the literature, see Karamardian and Schaible [5], for example. In [2], Crouzeix, et al. introduced the concepts of monotone plus mappings and proved the important role in the convergence of cutting-plane method for solving variational inequities. In [14], Zhu and Marcotte introduced the classes of generalized cocoercive mapping and related them to classes previously introduced. Zhu and Marcotte [15] investigate iterative schemes for solving nonlinear variational inequalities under cocoercive assumption.

Variational-like inequality problem (VLIP) or prevariational inequalities (PVI) is more general problem than VIP, which is first introduced by Parida et al. [9]. Invex monotonicity, which is a generalization of classical monotonicity, is investigated widely by many researchers for studying invex function, which is generalization of convex function [68, 12, 13], and solving VLIP [3,9-11]. Ruiz-Garzón et al. [10] introduce some generalized invex monotonicity which are also discussed in [13], mentioned as generalized invariant monotonicity.

The purpose of this paper is to introduce new classes of generalized invex monotone plus mappings and generalized invex cocoercive mappings and analyze their properties and relationships with respect to other concepts of invex monotonicity. Some examples, 
counterexamples, and theoretical results are offered. These concepts allow the development of the convergent algorithm to solving VLIP and characterization of the solution set of VLIP. This paper will be organized as follows: for easy of reference, the next section regroups all definitions of generalized monotonicity, invexity, and invex monotonicity required in our study; in Sections 3 and 4, we introduce the new class of generalized invex monotone plus mappings, and generalized invex cocoercive mappings respectively. We analyze the differential property of these new generalized invex monotone mappings in Section 5. We discuss the usefulness of the new concepts of generalized invex monotonicity for VLIP in Section 6. The concluding section concludes.

\section{Preliminaries}

Let $K$ be a nonempty subset of $\mathbb{R}^{n}, \eta: K \times K \rightarrow \mathbb{R}^{n}\left(K \subset \mathbb{R}^{n}\right)$, let $F$ be a vector-valued function from $K$ into $\mathbb{R}^{n}$, and let $f$ be a differentiable function from $K$ to $\mathbb{R}$.

Karamardian introduced some monotone mappings in [5]. In [2], some new monotonicity, such as monotone ${ }^{+}$and pseudomonotone ${ }^{+}$are introduced and applied to cutting-plane methods for solving variational inequalities.

Definition 2.1 [2]. $F$ is said to be

(i) monotone ${ }^{+}\left(\mathrm{M}^{+}\right)$on $K$ if it is monotone on $K$ and $\forall x, y \in K$,

$$
\langle F(y)-F(x), y-x\rangle=0 \Longrightarrow F(y)=F(x) ;
$$

(ii) monotone $_{*}^{+}\left(\mathrm{M}_{*}^{+}\right)$on $K$ if it is monotone on $K$ and $\forall x, y \in K$,

$$
\langle F(y), y-x\rangle=\langle F(x), y-x\rangle=0 \Longrightarrow F(y)=F(x) ;
$$

(iii) monotone $_{*}\left(\mathrm{M}_{*}\right)$ on $K$ if it is monotone on $K$ and $\forall x, y \in K$,

$$
\langle F(y), y-x\rangle=\langle F(x), y-x\rangle=0 \Longrightarrow \exists k>0 \text {, such that } F(y)=k F(x) \text {; }
$$

(iv) pseudomonotone $e^{+}\left(\mathrm{PM}^{+}\right)$on $K$ if it is pseudomonotone on $K$ and $\forall x, y \in K$,

$$
\langle F(y)-F(x), y-x\rangle=0 \Longrightarrow F(y)=F(x)
$$

(v) pseudomonotone ${ }_{*}^{+}\left(\mathrm{PM}_{*}^{+}\right)$on $K$ if it is pseudomonotone on $K$ and $\forall x, y \in K$,

$$
\langle F(y), y-x\rangle=\langle F(x), y-x\rangle=0 \Longrightarrow F(y)=F(x)
$$

(vi) pseudomonotone $*\left(\mathrm{PM}_{*}\right)$ on $K$ if it is pseudomonotone on $K$ and $\forall x, y \in K$,

$$
\langle F(y), y-x\rangle=\langle F(x), y-x\rangle=0 \Longrightarrow \exists k>0 \text {, such that } F(y)=k F(x) .
$$

Some relationships among the various generalized monotonicity can be represented by Figure 2.1 (see [2] for more details).

The cocoercive and generalized cocoercive mappings are introduced in [14]. The role of cocoercivity for solving variational inequalities is investigated in [15]. 


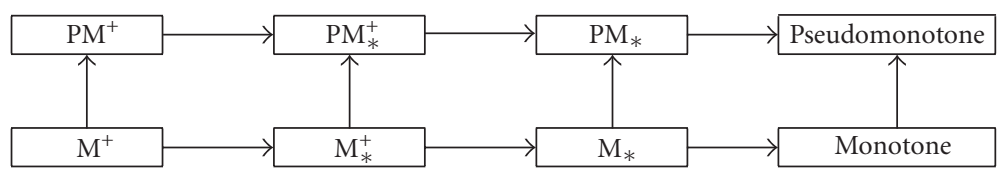

Figure 2.1. Relationships between the monotone plus classes.

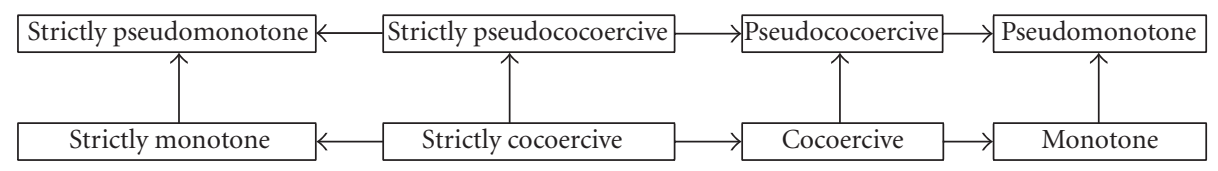

Figure 2.2. Relationships between generalized cocoercive mappings.

Definition 2.2 [14]. $F$ is said to be

(i) cocoercive on $K$ if there exists $\alpha>0$, for any $x, y \in K$,

$$
\langle F(y)-F(x), y-x\rangle \geq \alpha\|F(y)-F(x)\|^{2} ;
$$

(ii) strictly cocoercive on $K$ if there exists $\alpha>0$, for any distinct $x, y \in K$,

$$
\langle F(y)-F(x), y-x\rangle>\alpha\|F(y)-F(x)\|^{2} ;
$$

(iii) pseudococoercive on $K$ if there exists $\alpha>0$, for any distinct $x, y \in K$,

$$
\langle F(x), y-x\rangle \geq 0 \Longrightarrow\langle F(y), y-x\rangle \geq \alpha\|F(y)-F(x)\|^{2} ;
$$

(iv) strictly pseudococoercive on $K$ if there exists $\alpha>0$, for any distinct $x, y \in K$,

$$
\langle F(x), y-x\rangle \geq 0 \Longrightarrow\langle F(y), y-x\rangle>\alpha\|F(y)-F(x)\|^{2} .
$$

We can describe their relationships as shown in Figure 2.2 (see [14] for more details).

Invex function and generalized invex function are investigated by many authors, which are generalizations of convex function and generalized convex function $[6-8,12,13]$.

Definition 2.3 [10]. $f$ is said to be

(i) invex (IX) on $K$ with respect to $\eta$ if for any $x, y \in K$,

$$
f(y)-f(x) \geq\langle\nabla f(x), \eta(y, x)\rangle ;
$$

(ii) strictly invex (SIX) on $K$ with respect to $\eta$ if for any distinct $x, y \in K$,

$$
f(y)-f(x)>\langle\nabla f(x), \eta(y, x)\rangle ;
$$

(iii) strongly invex (SGIX) on $K$ with respect to $\eta$ if there exists $\alpha>0$, such that

$$
f(y)-f(x) \geq\langle\nabla f(x), \eta(y, x)\rangle+\alpha\|\eta(y, x)\|^{2}, \quad \forall x, y \in K ;
$$


4 New classes of generalized invex monotonicity

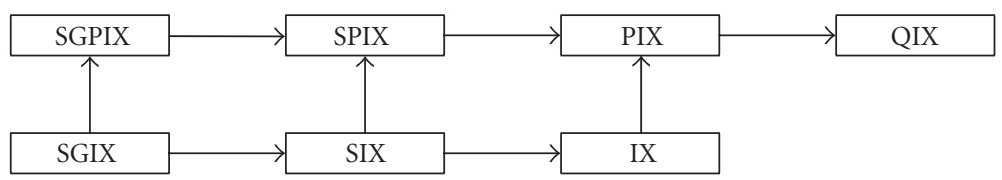

Figure 2.3. Relationships between the generalized invex functions.

(iv) pseudoinvex (PIX) on $K$ with respect to $\eta$ if for any $x, y \in K$,

$$
\langle\nabla f(x), \eta(y, x)\rangle \geq 0 \Longrightarrow f(y)-f(x) \geq 0 ;
$$

(v) strictly pseudoinvex (SPIX) on $K$ with respect to $\eta$ if for any distinct $x, y \in K$,

$$
\langle\nabla f(x), \eta(y, x)\rangle \geq 0 \Rightarrow f(y)-f(x)>0 ;
$$

(vi) strongly pseudoinvex (SGPIX) on $K$ with respect to $\eta$ if there exists $\alpha>0$, such that

$$
\langle\nabla f(x), \eta(y, x)\rangle \geq 0 \Rightarrow f(y) \geq f(x)+\alpha\|\eta(y, x)\|^{2}, \quad \forall x, y \in K
$$

(vii) quasi-invex (QIX) on $K$ with respect to $\eta$ if for any $x, y \in K$,

$$
f(y)-f(x) \leq 0 \Longrightarrow\langle\nabla f(x), \eta(y, x)\rangle \leq 0 .
$$

From the definitions, we can establish their relationships as shown in Figure 2.3.

In [10], the definitions of generalized invex monotonicity are offered, which generalize generalized monotonicity established by Karamardian [5].

Definition $2.4[10] . F$ is said to be

(i) invex monotone (IM) on $K$ with respect to $\eta$ if for any $x, y \in K$,

$$
\langle F(y)-F(x), \eta(y, x)\rangle \geq 0
$$

(ii) strictly invex monotone (SIM) on $K$ with respect to $\eta$ if for any distinct $x, y \in K$,

$$
\langle F(y)-F(x), \eta(y, x)\rangle>0
$$

(iii) strongly invex monotone (SGIM) on $K$ with respect to $\eta$ if there exists $\beta>0$, such that

$$
\langle F(y)-F(x), \eta(y, x)\rangle \geq \beta\|\eta(y, x)\|^{2}, \quad \forall x, y \in K
$$

(iv) pseudoinvex monotone (PIM) on $K$ with respect to $\eta$ if for any $x, y \in K$, we have

$$
\langle F(x), \eta(y, x)\rangle \geq 0 \Longrightarrow\langle F(y), \eta(y, x)\rangle \geq 0 ;
$$

(v) strictly pseudoinvex monotone (SPIM) on $K$ with respect to $\eta$ if for any distinct $x, y \in K$,

$$
\langle F(x), \eta(y, x)\rangle \geq 0 \Longrightarrow\langle F(y), \eta(y, x)\rangle>0
$$




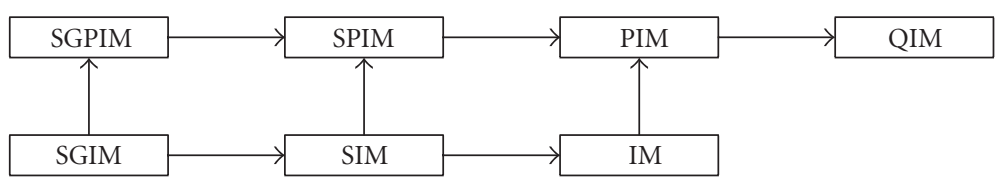

Figure 2.4. Relationships between the invex monotonicity classes.

(vi) strongly pseudoinvex monotone (SGPIM) on $K$ with respect to $\eta$ if there exists $\beta>$ 0 , such that

$$
\langle F(x), \eta(y, x)\rangle \geq 0 \Longrightarrow\langle F(y), \eta(y, x)\rangle \geq \beta\|\eta(y, x)\|^{2}, \quad \forall x, y \in K ;
$$

(vii) quasi-invex monotone (QIM) on $K$ if for any $x, y \in K$,

$$
\eta(y, x)^{T} F(x)>0 \Longrightarrow \eta(y, x)^{T} F(y) \geq 0 .
$$

From the definitions, their relationships are described as shown in Figure 2.4.

Remark 2.5. From the definition, we can see that every (generalized) monotone mapping is (generalized) invex monotone mapping with $\eta(x, y)=x-y$, but the converse is not necessarily true. Examples and counterexamples can be found in $[10,13]$.

Remark 2.6. When $\eta(x, y)+\eta(y, x)=0$, invariant monotonicity defined in [13] is equivalent to invex monotonicity.

\section{New class of generalized invex monotone mappings}

In this section, we will present the definitions of (pseudo) invex monotone plus mappings, and so forth, and discuss their relationships by examples and counterexamples.

\subsection{Invex monotone plus mappings}

Definition 3.1. $F$ is said to be

(i) invex monotone $e^{+}\left(\mathrm{IM}^{+}\right)$on $K$ with respect to $\eta$ if it is invex monotone on $K$ with respect to $\eta$ and, for any $x, y \in K$,

$$
\langle F(y)-F(x), \eta(y, x)\rangle=0 \Longrightarrow F(y)=F(x)
$$

(ii) invex monotone $e_{*}^{+}\left(\mathrm{IM}_{*}^{+}\right)$on $K$ with respect to $\eta$ if it is invex monotone on $K$ with respect to $\eta$ and, for any $x, y \in K$,

$$
\langle F(y), \eta(y, x)\rangle=\langle F(x), \eta(y, x)\rangle=0 \Longrightarrow F(y)=F(x) ;
$$

(iii) invex monotone ${ }_{*}\left(\mathrm{IM}_{*}\right)$ on $K$ with respect to $\eta$ if it is invex monotone on $K$ with respect to $\eta$ and, for any $x, y \in K$,

$$
\langle F(y), \eta(y, x)\rangle=\langle F(x), \eta(y, x)\rangle=0 \Longrightarrow \exists k>0, \quad \text { such that } F(y)=k F(x) .
$$


Remark 3.2. (i) Every $\mathrm{M}^{+}\left(\mathrm{M}_{*}^{+}, \mathrm{M}_{*}\right)$ mapping is $\mathrm{IM}^{+}\left(\mathrm{IM}_{*}^{+}, \mathrm{IM}_{*}\right)$ mapping with $\eta(x, y)=$ $x-y$, but the converse is not necessarily true.

(ii) According to the above definitions, we have $\mathrm{SIM} \Rightarrow \mathrm{IM}^{+} \Rightarrow \mathrm{IM}_{*}^{+} \Rightarrow \mathrm{IM}_{*} \Rightarrow \mathrm{IM}$, but the converse is not necessarily true.

Example 3.3. Let $F(x)=\left[\begin{array}{c}\sin x_{1} \\ \sin x_{2}\end{array}\right], \eta(x, y)=\left[\begin{array}{c}\sin x_{2}-\sin y_{2} \\ \sin y_{1}-\sin x_{1}\end{array}\right]$. Obviously, $F(x)$ is IM on $R^{2}$ with respect to $\eta$. Let $x=(\pi / 2, \pi / 2)^{T}, y=(-\pi / 2,-\pi / 2)^{T}$,

$$
\langle F(y), \eta(y, x)\rangle=\langle F(x), \eta(y, x)\rangle=0,
$$

but there is no $k>0$ such that $F(y)=k F(x)$. This implies that $F(x)$ is not $\mathrm{IM}_{*}$ on $R^{2}$ with respect to $\eta$.

Example 3.4. Let $F(x)=\left[\begin{array}{c}\sin x_{1} \\ \sin x_{2}\end{array}\right], \eta(x, y)=\left[\begin{array}{c}\sin x_{2}-\sin y_{2} \\ \sin y_{1}-\sin x_{1}\end{array}\right]$, and $K=(0, \pi) \times(0, \pi)$. By definition, $F(x)$ is $\mathrm{IM}_{*}$ on $K$ with respect to $\eta$. Let $x=(\pi / 2, \pi / 2)^{T}, y=(5 \pi / 6,5 \pi / 6)^{T}$, we have

$$
\langle F(y), \eta(y, x)\rangle=\langle F(x), \eta(y, x)\rangle=0,
$$

but $F(y) \neq F(x)$, which means $F(x)$ is not $\mathrm{IM}_{*}^{+}$on $K$ with $\eta$. Meanwhile, we have

$$
\langle F(y)-F(x), y-x\rangle=-\frac{\pi}{3}<0 .
$$

Therefore $F(x)$ is not $\mathrm{M}_{*}$ on $K$.

Example 3.5. Let $F(x)=\left[\begin{array}{c}\sin x_{2}-\sin x_{1} \\ -\sin x_{2}\end{array}\right], \eta(x, y)=\left[\begin{array}{c}\sin x_{2}-\sin y_{2} \\ \sin y_{1}-\sin x_{1}\end{array}\right]$, and $K=(0, \pi) \times(0, \pi)$. We have

$$
\langle F(y)-F(x), \eta(y, x)\rangle=\left(\sin y_{2}-\sin x_{2}\right)^{2}=0
$$

if and only if $\sin x_{2}=\sin y_{2}$. Furthermore, with the condition

$$
\langle F(x), \eta(y, x)\rangle=\left(\sin x_{2}-\sin x_{1}\right)\left(\sin x_{2}-\sin y_{2}\right)+\sin x_{2}\left(\sin x_{1}-\sin y_{1}\right)=0,
$$

we have $\sin x_{1}=\sin y_{1}$. It shows that $F(x)$ is $\mathrm{IM}_{*}^{+}$on $K$ with respect to $\eta$.

Let $x=(\pi / 2, \pi / 2)^{T}, y=(\pi / 6, \pi / 2)^{T}$, we have

$$
\langle F(y)-F(x), \eta(y, x)\rangle=0,
$$

but $F(y) \neq F(x)$. This implies $F(x)$ is not $\mathrm{IM}^{+}$on $K$ with respect to $\eta$. Meanwhile, $F(x)$ is not $\mathrm{M}_{*}^{+}$on $K$, since

$$
\langle F(y)-F(x), y-x\rangle=-\frac{\pi}{6}<0 .
$$

Example 3.6. Let $F(x)=\cos ^{2} x, \eta(x, y)=\sin ^{2} y-\sin ^{2} x$, and $K=(-\pi / 2, \pi / 2)$. Obviously, $F(x)$ is $\mathrm{IM}^{+}$on $K$ with respect to $\eta$, but not SIM on $K$ with $\eta$, since

$$
\langle F(y)-F(x), \eta(y, x)\rangle=0, \quad \text { if } x=-y \neq 0 .
$$


Meanwhile, $F(x)$ is not $\mathrm{M}^{+}$yet, since

$$
\langle F(y)-F(x), y-x\rangle=-\frac{\pi}{8}<0, \quad \text { if } x=0, y=\frac{\pi}{4} .
$$

\subsection{Pseudoinvex monotone plus mappings.}

Definition 3.7. $F$ is said to be

(i) pseudoinvex monotone ${ }^{+}\left(\mathrm{PIM}^{+}\right)$on $K$ with respect to $\eta$ if it is pseudoinvex monotone on $K$ with respect to $\eta$ and, for any $x, y \in K$,

$$
\langle F(y)-F(x), \eta(y, x)\rangle=0 \Longrightarrow F(y)=F(x)
$$

(ii) pseudoinvex monotone $e_{*}^{+}\left(\mathrm{PIM}_{*}^{+}\right)$on $K$ with respect to $\eta$ if it is pseudoinvex monotone on $K$ with respect to $\eta$ and, for any $x, y \in K$,

$$
\langle F(y), \eta(y, x)\rangle=\langle F(x), \eta(y, x)\rangle=0 \Longrightarrow F(y)=F(x)
$$

(iii) pseudoinvex monotone ${ }_{*}\left(\mathrm{PIM}_{*}\right)$ on $K$ with respect to $\eta$ if it is pseudoinvex monotone on $K$ with respect to $\eta$ and, for any $x, y \in K$,

$$
\langle F(y), \eta(y, x)\rangle=\langle F(x), \eta(y, x)\rangle=0 \Longrightarrow \exists k>0, \quad \text { such that } F(y)=k F(x) .
$$

Remark 3.8. (i) Every $\mathrm{PM}^{+}\left(\mathrm{PM}_{*}^{+}, \mathrm{PM}_{*}\right)$ mapping is $\mathrm{PIM}^{+}\left(\mathrm{PIM}_{*}^{+}, \mathrm{PIM}_{*}\right)$ mapping with $\eta(x, y)=x-y$, but the converse is not necessarily true.

(ii) According to the above definitions, we have $\mathrm{PIM}^{+} \Rightarrow \mathrm{PIM}_{*}^{+} \Rightarrow \mathrm{PIM}_{*} \Rightarrow \mathrm{PIM}$ and $\mathrm{SPIM} \Rightarrow \mathrm{PIM}_{*}^{+}$, but the converse is not necessarily true.

(iii) Obviously, we have the relationships, $\mathrm{IM}^{+} \Rightarrow \mathrm{PIM}^{+}, \mathrm{IM}_{*}^{+} \Rightarrow \mathrm{PIM}_{*}^{+}$, and $\mathrm{IM}_{*} \Rightarrow$ $\mathrm{PIM}_{*}$, but the converse is not true.

Example 3.9. Let $F(x)=\left[\begin{array}{l}\sin x_{1} \\ \sin x_{2}\end{array}\right], \eta(x, y)=\left[\begin{array}{c}\sin x_{2}-\sin y_{2} \\ 0\end{array}\right]$, and $K=(0, \pi) \times(0, \pi)$. Obviously, $F(x)$ is PIM on $K$ with respect to $\eta$. Let $x=(\pi / 2, \pi / 2)^{T}, y=(\pi / 3, \pi / 2)^{T}$, we have

$$
\langle F(y), \eta(y, x)\rangle=\langle F(x), \eta(y, x)\rangle=0,
$$

but there is no $k>0$ such that $F(y)=k F(x)$. This implies that $F(x)$ is not $\mathrm{PIM}_{*}$ on $K$ with respect to $\eta$.

Example 3.10. Let $F(x)=\left[\sin x_{1} / \sin ^{2} x_{2}, 1 / \sin x_{2}\right]^{T}, \eta(x, y)=\left[\begin{array}{l}\sin x_{2}-\sin y_{2} \\ \sin y_{1}-\sin x_{1}\end{array}\right]$, and $K=$ $(0, \pi) \times(0, \pi)$. From the definition, we know $F(x)$ is $\operatorname{PIM}_{*}$ on $K$ with respect to $\eta$. Let $x=(\pi / 2, \pi / 2)^{T}, y=(\pi / 4, \pi / 4)^{T}$, we have

$$
\langle F(y), \eta(y, x)\rangle=\langle F(x), \eta(y, x)\rangle=0,
$$

but $F(y) \neq F(x)$, which means $F(x)$ is not $\mathrm{PIM}_{*}^{+}$on $K$ with $\eta$.

Furthermore, let $x=(\pi / 2, \pi / 2)^{T}, y=(\pi / 4,5 \pi / 6)^{T}$, we have

$$
\langle F(y)-F(x), \eta(y, x)\rangle=\frac{(3-3 \sqrt{2})}{2}<0 .
$$


8 New classes of generalized invex monotonicity

Therefore $F(x)$ is not $\mathrm{IM}_{*}$ on $K$ with $\eta$. Meanwhile, $F(x)$ is not $\mathrm{PM}_{*}$ on $K$, since

$$
\langle F(x), y-x\rangle=\frac{\pi}{12}>0, \quad\langle F(y), y-x\rangle=\frac{(4-3 \sqrt{2}) \pi}{6}<0 .
$$

Example 3.11. Let $F(x)=\left[\begin{array}{c}\sin x_{1} \\ \sin x_{2}\end{array}\right], \eta(x, y)=\left[\begin{array}{c}\left(\sin x_{2}-\sin y_{2}\right)^{2} \\ \left(\sin y_{1}-\sin x_{1}\right)^{2}\end{array}\right]$, and $K=(0, \pi) \times(0, \pi)$. It is easy to proof that $F(x)$ is $\mathrm{PIM}_{*}^{+}$on $K$ with respect to $\eta$. Let $x=(\pi / 2,5 \pi / 6)^{T}, y=(5 \pi / 6, \pi / 6)^{T}$, we have

$$
\langle F(y)-F(x), \eta(y, x)\rangle=0,
$$

but $F(y) \neq F(x)$. This implies $F(x)$ is not $\mathrm{PIM}^{+}$on $K$ with respect to $\eta$. Furthermore, we can see that $F(x)$ is not $\mathrm{PM}_{*}^{+}$on $K$, since

$$
\langle F(x), y-x\rangle=0, \quad\langle F(y), y-x\rangle=-\frac{\pi}{6}<0 .
$$

On the other hand, if we set $x=(\pi / 2, \pi / 6)^{T}, y=(\pi / 3, \pi / 2)^{T}$, we have

$$
\langle F(y)-F(x), \eta(y, x)\rangle=\frac{(1-\sqrt{3})(2-\sqrt{3})}{8}<0,
$$

which shows that $F(x)$ is not $\mathrm{IM}_{*}^{+}$on $K$ with respect to $\eta$.

Example 3.12. Let $F(x)=\left[\begin{array}{c}\sin x_{1} \\ 1\end{array}\right], \eta(x, y)=\left[\begin{array}{c}\sin y_{1}-\sin x_{1} \\ 0\end{array}\right]$, and $K=(0, \pi) \times(0, \pi)$. Obviously, $F(x)$ is $\mathrm{PIM}^{+}$, but not $\mathrm{IM}^{+}$, on $K$ with respect to $\eta$, since

$$
\langle F(y)-F(x), \eta(y, x)\rangle=-\left(\sin y_{1}-\sin x_{1}\right)^{2}<0, \quad \text { if } x_{1} \neq y_{1}
$$

Furthermore, $F(x)$ is not $\mathrm{PM}^{+}$, since $x=(\pi / 2, \pi / 2)^{T}, y=(3 \pi / 4, \pi / 4)^{T}$, we have

$$
\langle F(x), y-x\rangle=0, \quad\langle F(y), y-x\rangle=(\sqrt{2}-2) \frac{\pi}{8}<0 .
$$

\section{New class of generalized invex cocoercive mappings}

In this section, we will firstly present the definitions of generalized invex cocoercive mappings, which generalize cocoercive mappings. Then their relationships are discussed by examples and counterexamples.

\subsection{Invex cocoercive and invex Lipschitz continuous.}

Definition 4.1. $F$ is said to be invex cocoercive on $K$ with respect to $\eta$ if there exists $\alpha>0$, for any $x, y \in K$,

$$
\langle F(y)-F(x), \eta(y, x)\rangle \geq \alpha\|F(y)-F(x)\|^{2} .
$$

Every cocoercive mapping is invex cocoercive with $\eta(x, y)=x-y$, but the converse is not necessarily true. 
Example 4.2 [12, Reconstruct Example 1.4]. Let $F(x)=-|x|, x \in R$,

$$
\eta(x, y)= \begin{cases}y-x, & \text { if } x \geq 0, y \geq 0, \\ x-y, & \text { if } x \leq 0, y \leq 0, \\ x+y, & \text { if } x \leq 0, y \geq 0, \\ -x-y, & \text { if } x \geq 0, y \leq 0 .\end{cases}
$$

It is easy to proof that $F(x)$ is invex cocoercive with $\eta$, but not cocoercive, since

$$
\langle F(y)-F(x), y-x\rangle=-(y-x)^{2}<0, \quad \text { if } x>0, y>0 \text {, and } x \neq y \text {. }
$$

Remark 4.3. An invex cocoercive mapping is $\mathrm{IM}^{+}$with the same $\eta$, as a comparison of (3.1) and (4.1), but the converse is not true.

Example 4.4. Let $F(x)=\cos x, \eta(x, y)=\sin ^{2} y-\sin ^{2} x$, and $K=(0, \pi / 2)$. Obviously, $F(x)$ is $\mathrm{IM}^{+}$on $K$ with respect to $\eta$, but not invex cocoercive, on $K$ with $\eta$, since there is no $\alpha>0$, for any $x, y \in(0, \pi / 2)$, such that

$$
\begin{aligned}
\langle F(y)-F(x), \eta(y, x)\rangle & =(\cos y+\cos x)(\cos y-\cos x)^{2} \\
& \geq \alpha(\cos y-\cos x)^{2}=\alpha\|F(y)-F(x)\|^{2}
\end{aligned}
$$

Definition 4.5. $F$ is said to be invex Lipschitz continuous on $K$ with respect to $\eta$ if there exists $L>0$, for any $x, y \in K$,

$$
\|F(y)-F(x)\| \leq L\|\eta(y, x)\|
$$

Every Lipschitz continuous mapping is invex Lipschitz continuous with $\eta(x, y)=x-$ $y$, but the converse is not necessarily true.

Example 4.6. Let $F(x)=\left[\begin{array}{cc}0 & 1 \\ -1 & 0\end{array}\right]\left[\begin{array}{l}x_{1}^{2} \\ x_{2}^{2}\end{array}\right], \eta(x, y)=\left[\begin{array}{c}x_{1}^{2}-y_{1}^{2} \\ x_{2}^{2}-y_{2}^{2}\end{array}\right]$. We can see that $F(x)$ is not Lipschitz continuous and invex cocoercive, though it is invex Lipschitz continuous and IM with respect to $\eta(x, y)$ on $R^{2}$.

The sum of invex cocoercive mappings with the same $\eta$ is invex cocoercive. The next proposition shows that invex Lipschitz continuous and SGIM can ensure invex cocoercive.

Proposition 4.7. With respect to $\eta$, let $F$ be invex Lipschitz continuous with constant $L$, and SGIM with modulus $\beta$ on $K$. Then with the same $\eta, F$ is invex cocoercive with modulus $\beta / L^{2}$ on $K$.

Proof. This is straightforward from (2.20) and (4.5).

The converse of Proposition 4.7 is not true, since a constant mapping is trivially invex cocoercive but clearly not SGIM. On the other hand, invex cocoercive mapping is invex 
Lipschitz continuous with the same $\eta$, since from the Schwarz inequality and (4.1), there exists

$$
\|F(y)-F(x)\|\|\| \eta(y, x)\|\geq\langle F(y)-F(x), \eta(y, x)\rangle \geq \alpha\| F(y)-F(x) \|^{2},
$$

but the converse is not true as the Example 4.6 is a counterexample.

\subsection{Strictly invex cocoercive}

Definition 4.8. $F$ is said to be strictly invex cocoercive on $K$ with respect to $\eta$ if there exists $\alpha>0$, for every pair of distinct $x, y \in K$,

$$
\langle F(y)-F(x), \eta(y, x)\rangle>\alpha\|F(y)-F(x)\|^{2} .
$$

Every strictly cocoercive mapping is strictly invex cocoercive mapping with $\eta(x, y)=$ $x-y$, but the converse is not necessarily true.

Example 4.9. Let $F(x)=-\sin x, x \in(\pi / 4,3 \pi / 4), \eta(x, y)=\cos ^{2} x-\cos ^{2} y$. Then $F(x)$ is strictly invex cocoercive with $\eta(x, y)$, since if $x \neq y$, we have

$$
\langle F(y)-F(x), \eta(y, x)\rangle=(\sin x+\sin y)(\sin x-\sin y)^{2}>\sqrt{2}\|F(y)-F(x)\|^{2} .
$$

But $F(x)$ is not strictly cocoercive, since

$$
\langle F(y)-F(x), y-x\rangle=(\sqrt{2}-2) \pi / 8<0, \quad \text { if } x=\frac{\pi}{2}, y=\frac{\pi}{4} .
$$

Remark 4.10. A strictly invex cocoercive mapping is SIM and invex cocoercive with the same $\eta$, as a comparison of (2.19), (4.1), and (4.7), but the converse is not true.

Example 4.11. Let $F(x)=\left[\begin{array}{cc}0 & 1 \\ -1 & 0\end{array}\right]\left[\begin{array}{l}x_{1}^{2} \\ x_{2}^{2}\end{array}\right]$, we have

(i) $F(x)$ is SIM, but not strictly invex cocoercive, with respect to $\eta(x, y)=\left[\begin{array}{l}x_{2}-y_{2} \\ y_{1}-x_{1}\end{array}\right]$ on $R_{+}^{2}=\{(x, y) \in R \times R \mid x \geq 0, y \geq 0\}$.

(ii) $F(x)$ is invex cocoercive, but not strictly invex cocoercive, with respect to $\eta(x, y)=$ $\left[\begin{array}{l}x_{2}^{2}-y_{2}^{2} \\ y_{1}^{2}-x_{1}^{2}\end{array}\right]$ on $R^{2}$. Since if $x=-y$, there does not exist any $\alpha>0$, such that

$$
0=\langle F(y)-F(x), \eta(y, x)\rangle>\alpha\|F(y)-F(x)\|^{2}=0 .
$$

The sum of a strictly invex cocoercive mapping and an invex cocoercive mapping with the same $\eta$ is strictly invex cocoercive. The next proposition shows that the invex Lipschitz continuous and SGIM can ensure strictly invex cocoercive.

Proposition 4.12. With respect to $\eta$, let nonconstant mapping $F$ be invex Lipschitz continuous with constant $L$, and SGIM with modulus $\beta$ on $K$. Then with the same $\eta, F$ is strictly invex cocoercive with modulus $\beta / L^{2}$ on $K$.

Proof. This is straightforward from (2.20), (4.5), and (4.7).

The converse of Proposition 4.12 is not true, since a strictly invex cocoercive mapping is not necessarily SGIM according to the following example. 
Example 4.13. Let $F(x)=\sin ^{3} x, \eta(x, y)=\sin x-\sin y, x, y \in[-\pi / 2, \pi / 2]$, we have

$$
0<\sin ^{2} x+\sin x \sin y+\sin ^{2} y<3, \quad \forall x, y \in[-\pi / 2, \pi / 2], x \neq y,
$$

thus

$$
\begin{aligned}
\langle F(y)-F(x), \eta(y, x)\rangle & =(\sin y-\sin x)^{2}\left(\sin ^{2} x+\sin x \sin y+\sin ^{2} y\right) \\
& >\frac{1}{3}(\sin y-\sin x)^{2}\left(\sin ^{2} x+\sin x \sin y+\sin ^{2} y\right)^{2} \\
& =\frac{1}{3}\|F(y)-F(x)\|^{2} .
\end{aligned}
$$

Therefore, $F(x)$ is strictly invex cocoercive with $\eta$. However it is not SGIM with $\eta$, since there does not exist any $\beta>0$, such that

$$
\sin ^{2} x+\sin x \sin y+\sin ^{2} y \geq \beta .
$$

\subsection{Pseudoinvex cocoercive}

Definition 4.14. $F$ is said to be pseudoinvex cocoercive on $K$ with respect to $\eta$ if there exists $\alpha>0$, for every pair of distinct $x, y \in K$,

$$
\langle F(x), \eta(y, x)\rangle \geq 0 \Longrightarrow\langle F(y), \eta(y, x)\rangle \geq \alpha\|F(y)-F(x)\|^{2} .
$$

Every pseudococoercive mapping is pseudoinvex cocoercive mapping with $\eta(x, y)=$ $x-y$, but the converse is not necessarily true.

Example 4.15. Let

$$
F(x)=\left\{\begin{array}{ll}
-e^{x} & \text { if } x>0 \\
0 & \text { if } x \leq 0
\end{array}, \quad \eta(x, y)=e^{y}-e^{x}\right.
$$

Obviously, $F(x)$ is pseudoinvex cocoercive with $\eta$, but not pseudococoercive, since

$$
\langle F(x), y-x\rangle=0, \quad\langle F(y), y-x\rangle=-y e^{y}<0, \quad \text { if } x=0, y>0 .
$$

Remark 4.16. An invex cocoercive mapping is pseudoinvex cocoercive and a pseudoinvex cocoercive mapping is $\mathrm{PIM}_{*}^{+}$(but not necessarily $\mathrm{PIM}^{+}$) with the same $\eta$, as a comparison of (3.14), (4.1), and (4.14), but the converse is not true.

Example 4.17. Let $F(x)=\left(1+e^{x}\right)^{-1}, \eta(x, y)=e^{x}-e^{y}, x, y \in R$. For every pair of distinct $x, y,\langle F(x), \eta(y, x)\rangle \geq 0$ implies $y>x$, thus

$$
\langle F(y), \eta(y, x)\rangle=\frac{e^{y}-e^{x}}{1+e^{y}} \geq \frac{\left(e^{y}-e^{x}\right)^{2}}{\left(1+e^{x}\right)^{2}\left(1+e^{y}\right)^{2}}=\|F(y)-F(x)\|^{2},
$$

which means that $F(x)$ is pseudoinvex cocoercive with $\eta(x, y)$. But $F(x)$ fails to be invex cocoercive, even IM with $\eta(x, y)$, since if $x \neq y$, we have

$$
\langle F(y)-F(x), \eta(y, x)\rangle=-\left(e^{y}-e^{x}\right)^{2}\left(1+e^{x}\right)^{-1}\left(1+e^{y}\right)^{-1}<0 .
$$


Example 4.18 (see [13, Example 4.2]). Let $F(x)=\cos ^{2} x, \eta(x, y)=\cos y-\cos x, x, y \in$ $(-\pi / 2, \pi / 2)$. Clearly, $F(x)$ is $\mathrm{PIM}_{*}^{+}$and $\mathrm{PIM}^{+}$with $\eta(x, y)$. Assume $F(x)$ be pseudoinvex cocoercive with $\eta(x, y)$, let $y>x=0$, we have $\langle F(x), \eta(y, x)\rangle \geq 0$, and

$$
\langle F(y), \eta(y, x)\rangle=\cos ^{2} y(1-\cos y) \geq \alpha\left(\cos ^{2} y-1\right)^{2}=\alpha\|F(y)-F(x)\|^{2} .
$$

Taking limit $y \rightarrow \pi / 2$ in above inequality, we obtain a contradiction: $0 \geq \alpha$, which means that $F(x)$ is not pseudoinvex cocoercive.

The next proposition can be straightforward from (2.23), (4.5), and (4.14).

Proposition 4.19. With respect to $\eta$, let $F$ be invex Lipschitz continuous with constant $L$, and SGPIM with modulus $\beta$ on $K$. Then with the same $\eta, F$ is pseudoinvex cocoercive with modulus $\beta / L^{2}$ on $K$.

The converse of Proposition 4.19 is not true, since pseudoinvex cocoercive mapping is not necessary SGPIM. For example, let $x=0, y>0$ in Example 4.17, there exists $\langle F(x), \eta(y, x)\rangle \geq$ 0 , but there does not exist any $\alpha>0$, such that

$$
\langle F(y), \eta(y, x)\rangle=\left(e^{y}-1\right)\left(1+e^{y}\right)^{-1} \geq \alpha\left(e^{y}-1\right)^{2}=\alpha\|\eta(y, x)\|^{2},
$$

which shows that $F(x)$ is not SGPIM with $\eta$ on $R$.

\subsection{Strictly pseudoinvex cocoercive}

Definition 4.20. $F$ is said to be strictly pseudoinvex cocoercive on $K$ with respect to $\eta$ if there exists $\alpha>0$, for every pair of distinct $x, y \in K$,

$$
\langle F(x), \eta(y, x)\rangle \geq 0 \Longrightarrow\langle F(y), \eta(y, x)\rangle>\alpha\|F(y)-F(x)\|^{2} .
$$

Every strictly pseudococoercive mapping is strictly pseudoinvex cocoercive mapping with $\eta(x, y)=x-y$, but the converse is not necessarily true.

Example 4.21. Let $F(x)=-x, \eta(x, y)=a(y-x), x \leq 0, y \leq 0, a \geq 1$. Obviously, $F(x)$ is strictly pseudoinvex cocoercive with $\eta$, but not strictly pseudococoercive, since

$$
\langle F(y), y-x\rangle=-y^{2}<0, \quad \text { if } x=0, y<0 .
$$

Remark 4.22. A strictly pseudoinvex cocoercive mapping is pseudoinvex cocoercive and SPIM with the same $\eta$, as a comparison of (2.22), (4.14), and (4.21), but the converse is not true.

For example, $F(x)$ presented in Example 4.15 is pseudoinvex cocoercive with $\eta$, but not strictly pseudoinvex cocoercive, since $F(x)=F(y)=0$, whenever $x \leq 0, y \leq 0, x \neq y$.

We can see that $F(x)=\cos ^{2} x$ is SPIM, but not strictly pseudoinvex cocoercive with $\eta(x, y)=\cos y-\cos x$ on $[0, \pi / 2)$ according to Example 4.18 .

Similarly, invex Lipschitz continuous and SGPIM mapping are strictly pseudoinvex cocoercive, but the converse is not true.

Remark 4.23. A strictly pseudoinvex cocoercive mapping is not necessarily invex cocoercive, IM and SGPIM with the same $\eta$. 
Example 4.24. Let $F(x)=x, \eta(x, y)=y^{2}-x^{2}, x, y \in[1,+\infty)$. For every pair of distinct $x$, $y,\langle F(x), \eta(y, x)\rangle \geq 0$ implies $x>y$, thus

$$
\langle F(y), \eta(y, x)\rangle=y\left(x^{2}-y^{2}\right)>(y-x)^{2}=\|F(y)-F(x)\|^{2},
$$

which means that $F(x)$ is strictly pseudoinvex cocoercive with $\eta$. But $F(x)$ is neither invex cocoercive nor IM, since

$$
\langle F(y)-F(x), \eta(y, x)\rangle=-(y+x)(y-x)^{2}<0, \quad \text { if } x \neq y .
$$

Furthermore, $F(x)$ is not SGPIM with $\eta$, since there does not exist any $\beta>0$, such that $\langle F(y), \eta(y, x)\rangle=y\left(x^{2}-y^{2}\right) \geq \beta\left(x^{2}-y^{2}\right)^{2}=\beta\|\eta(y, x)\|^{2}$.

\section{Differential property}

In this section, we discuss the differential characterizations of the generalized invex monotonicity classes previously introduced. Throughout this section, we assume that function $f$ and mapping $F$ are continuously differentiable on set $K$.

In [10], the authors established the following relationships: if $f$ is IX (SIX, SGIX, PIX, SPIX, QIX) on $K$ with respect to $\eta$ skew, that is, $\eta(x, y)+\eta(y, x)=0$, then $\nabla f$ is IM (SIM, SGIM, PIM, SPIM, QIM). Furthermore, if $K$ is an open convex set, $\eta$ is linear in the first argument and $\eta(y, x)>0, \forall x, y \in K$, then $\nabla f$ is IM (PIM, SPIM) conversely implies $f$ is IX (PIX, SPIX), with respect to $\eta$. In this section, we firstly present that $f$ be IX (PIX) implies $\nabla f$ be $\mathrm{IM}_{*}\left(\mathrm{PIM}_{*}\right)$. Then we discuss the condition for (pseudo) IM to be (pseudo) $\mathrm{IM}_{*}, \mathrm{IM}_{*}^{+}$, and $\mathrm{IM}^{+}$. Firstly, we need the following assumption.

Let $\eta: K \times K \rightarrow R^{n}$, for any $x, y, z \in K$, such that the following hold.

Assumption 5.1. $\eta$ is skew, that is, $\eta(x, y)+\eta(y, x)=0$.

Assumption 5.2. $\eta(x, y)+\eta(y, z)=\eta(x, z)$.

Assumption 5.3. $\eta$ is continuous and linear in the first argument.

Lots of mappings satisfy Assumptions 5.1, 5.2, and 5.3, for example, the mappings $\eta(x, y)$ in Examples 4.2 and 4.21, especially $\eta(x, y)=x-y$ as well. Examples 3.3-3.6, 3.9, 3.10 suit Assumptions 5.1, 5.2.

Proposition 5.4. Let $K$ be an open convex set, $\eta$ satisfies Assumptions 5.1, 5.3. If $f$ is PIX on $K$ with respect to $\eta$, then $\nabla f$ is $P_{I} M_{*}$ on $K$ with the same $\eta$.

Proof. Let $u, v \in K, u \neq v$, such that $\nabla f(u) \neq 0, \nabla f(v) \neq 0$, and

$$
\langle\nabla f(u), \eta(u, v)\rangle=\langle\nabla f(v), \eta(u, v)\rangle=0 .
$$

Since $f$ is pseudoinvex and $\eta$ is skew, we have $f(u)=f(v)$. Take any vector $w \in R^{n}$ such that $\langle\nabla f(u), w\rangle<0$, we need to proof that $\langle\nabla f(v), w\rangle<0$.

If $\langle\nabla f(v),-w\rangle \leq 0$. Since $K$ is open, there exists $t>0$, such that $a=u+t w, b=v-$ $t w \in K$ and $f(a)<f(u)=f(v), f(b) \leq f(v)=f(u)$. We have

$$
\langle\nabla f(v), \eta(a, v)\rangle<0, \quad\langle\nabla f(v), \eta(b, v)\rangle \leq 0,
$$


where the first inequality is true for pseudoinvexity of $f$, the second holds for pseudoinvexity impling quasi invexity. From (5.2) and the fact that $\eta$ is linear in first argument, we have

$$
0>\langle\nabla f(v), \eta(a, v)+\eta(b, v)\rangle=\langle\nabla f(v), \eta(a+b, v)\rangle=\langle\nabla f(v), \eta(u, v)+\eta(v, v)\rangle=0 .
$$

We obtain a contradiction. It means $\langle\nabla f(v), w\rangle<0$. Permutate $u$ and $v$, repeat the argument. We obtain that $\langle\nabla f(u), w\rangle<0$ if and only if $\langle\nabla f(v), w\rangle<0$. Hence, there must exist a positive number $k$ such that $\nabla f(u)=k \nabla f(v)$, that is, $\nabla f$ is $\operatorname{PIM}_{*}$.

Corollary 5.5. Let $K$ be an open convex set, $\eta$ satisfies Assumptions 5.1, 5.3. If $f$ is invex on $K$ with respect to $\eta$, then $\nabla f$ is $I M_{*}$ on $K$ with the same $\eta$.

Now, we present the conditions for (pseudo) IM mappings to be (pseudo) IM $M_{*}, I M_{*}^{+}, I M^{+}$ mappings. The following lemma is required.

Lemma 5.6. Let $K$ be an open convex set, let $F$ be differentiable and PIM on $K$, $\eta$ satisfies Assumptions 5.1, 5.3. If $a, b \in K$ and $\langle F(a), \eta(b, a)\rangle=\langle F(b), \eta(b, a)\rangle=0$. Then for any $x_{\lambda}=a+\lambda(b-a), \lambda \in[0,1]$, there exists $\left\langle F\left(x_{\lambda}\right), \eta(b, a)\right\rangle=\left\langle F^{\prime}\left(x_{\lambda}\right)(b-a), \eta(b, a)\right\rangle=0$.

Proof. Since $\eta$ is skew and linear in first argument, we have

$$
\eta\left(x_{\lambda}, a\right)=\eta((1-\lambda) a+\lambda b, a)=(1-\lambda) \eta(a, a)+\lambda \eta(b, a)=\lambda \eta(b, a) .
$$

From (5.4) and pseudoinvex monotonicity of $F$, we have

$$
\left\langle F(a), \eta\left(x_{\lambda}, a\right)\right\rangle=\lambda\langle F(a), \eta(b, a)\rangle=0 \Longrightarrow\left\langle F\left(x_{\lambda}\right), \eta\left(x_{\lambda}, a\right)\right\rangle=\lambda\left\langle F\left(x_{\lambda}\right), \eta(b, a)\right\rangle \geq 0 .
$$

Symmetrically, we obtain $(1-\lambda)\left\langle F\left(x_{\lambda}\right), \eta(a, b)\right\rangle \geq 0, \forall \lambda \in[0,1]$, by exchanging $a$ and $b$. Since $\eta$ is skew, we have $g(\lambda)=\left\langle F\left(x_{\lambda}\right), \eta(b, a)\right\rangle=0, \forall \lambda \in[0,1]$. Thus $g^{\prime}(\lambda)=\left\langle F^{\prime}\left(x_{\lambda}\right)(b-\right.$ a), $\eta(b, a)\rangle=0$.

Proposition 5.7. Let $K$ be an open convex set, let $F$ be differentiable and (pseudo) IM on $K, \eta$ satisfies Assumptions 5.1, 5.2, 5.3. For any $x, y \in K$, assume that there holds

$$
\begin{gathered}
F(x)=0, \quad F(y) \neq 0 \Longrightarrow\langle F(y), \eta(y, x)\rangle>0 . \\
F(x) \neq 0, \quad\langle F(x), \eta(y, x)\rangle=\left\langle F^{\prime}(x)(y-x), \eta(y, x)\right\rangle=0 \Longrightarrow \exists t, \\
\text { such that } F^{\prime}(x)(y-x)=t F(x) .
\end{gathered}
$$

Then $F$ is (pseudo) $I M_{*}$ on $K$. Furthermore, if $F$ is affine, the condition is necessary as well.

Proof. Assume distinct $a, b \in K$, such that $\langle F(a), \eta(b, a)\rangle=\langle F(b), \eta(b, a)\rangle=0$. Set $x_{\lambda}=$ $a+\lambda(b-a), \lambda \in[0,1]$. From Lemma 5.6, we have

$$
\left\langle F\left(x_{\lambda}\right), \eta(b, a)\right\rangle=\left\langle F^{\prime}\left(x_{\lambda}\right)(b-a), \eta(b, a)\right\rangle=0 .
$$


If $F\left(x_{\lambda_{1}}\right)=0, \lambda_{1} \in[0,1]$, then for any $\lambda_{2} \in[0,1], \lambda_{2} \neq \lambda_{1}$, we have

$$
\left\langle F\left(x_{\lambda_{2}}\right), \eta\left(x_{\lambda_{2}}, x_{\lambda_{1}}\right)\right\rangle=\left\langle F\left(x_{\lambda_{2}}\right), \eta\left(x_{\lambda_{2}}, a\right)-\eta\left(x_{\lambda_{1}}, a\right)\right\rangle=\left(\lambda_{2}-\lambda_{1}\right)\left\langle F\left(x_{\lambda_{2}}\right), \eta(b, a)\right\rangle=0 .
$$

Considering (5.6), we can infer that $F\left(x_{\lambda}\right)=0$ for all $\lambda \in[0,1]$. In particular, $F(b)=$ $k F(a)=0$ for any $k>0$, that is, $F$ is (pseudo) $\mathrm{IM}_{*}$.

On the other hand, assume that $F\left(x_{\lambda}\right) \neq 0$ for all $\lambda \in[0,1]$. Since $\eta\left(x_{\lambda}, a\right)=\lambda \eta(b, a)$, we have

$$
\left\langle F\left(x_{\lambda}\right), \eta\left(x_{\lambda}, a\right)\right\rangle=\left\langle F^{\prime}\left(x_{\lambda}\right)\left(x_{\lambda}-a\right), \eta\left(x_{\lambda}, a\right)\right\rangle=0 .
$$

Hence by (5.7), there exists $t_{\lambda}$ such that $F^{\prime}\left(x_{\lambda}\right)\left(x_{\lambda}-a\right)=t_{\lambda} F\left(x_{\lambda}\right)$.

Set $G(x)=F(x) /\|F(x)\|$, where $\|F(x)\| \neq 0$, we obtain

$$
\begin{aligned}
G^{\prime}\left(x_{\lambda}\right)\left(x_{\lambda}-a\right) & =\left(1-\frac{F\left(x_{\lambda}\right) F^{T}\left(x_{\lambda}\right)}{\left\|F\left(x_{\lambda}\right)\right\|^{2}}\right) \frac{F^{\prime}\left(x_{\lambda}\right)\left(x_{\lambda}-a\right)}{\left\|F\left(x_{\lambda}\right)\right\|} \\
& =\left(1-\frac{F\left(x_{\lambda}\right) F^{T}\left(x_{\lambda}\right)}{\left\|F\left(x_{\lambda}\right)\right\|^{2}}\right) \frac{t_{\lambda} F\left(x_{\lambda}\right)}{\left\|F\left(x_{\lambda}\right)\right\|}=0 .
\end{aligned}
$$

From which we have $G\left(x_{\lambda}\right)=G(a)=G(b)$. The result is proved.

Furthermore, if $F(x)=A x+c$ be affine and (pseudo) $\mathrm{IM}_{*}$. It is obvious for (5.6) to be true. Now, let $x, y \in K$ such that

$$
\langle F(x), \eta(y, x)\rangle=\left\langle F^{\prime}(x)(y-x), \eta(y, x)\right\rangle=0,
$$

that is,

$$
\langle A x+c, \eta(y, x)\rangle=\langle A(y-x), \eta(y, x)\rangle=0,
$$

or equivalently:

$$
\langle A x+c, \eta(y, x)\rangle=\langle A[x+(y-x)]+c, \eta(y, x)\rangle=0 .
$$

Since $F$ is (pseudo) $\mathrm{IM}_{*}$, there exists a positive number $k$ such that $A[x+(y-x)]+c=$ $k(A x+c)$. From which we obtain $F^{\prime}(x)(y-x)=(k-1) F(x)$.

Proposition 5.8. Let $K$ be an open convex set, let $F$ be differentiable and (pseudo) IM on $K$, $\eta$ satisfies Assumptions 5.1, 5.3. For any $x, y \in K$, assume that there holds:

$$
\langle F(x), \eta(y, x)\rangle=\left\langle F^{\prime}(x)(y-x), \eta(y, x)\right\rangle=0 \Longrightarrow F^{\prime}(x)(y-x)=0 .
$$

Then $F$ is (pseudo) $I M_{*}^{+}$on K. Furthermore, if $F$ is affine, the condition is necessary as well. Proof. Assume distinct $a, b \in K$, such that $\langle F(a), \eta(b, a)\rangle=\langle F(b), \eta(b, a)\rangle=0$. Set $x_{\lambda}=$ $a+\lambda(b-a), \lambda \in[0,1]$. From Lemma 5.6, we have

$$
\left\langle F\left(x_{\lambda}\right), \eta(b, a)\right\rangle=\left\langle F^{\prime}\left(x_{\lambda}\right)(b-a), \eta(b, a)\right\rangle=0 .
$$


Since $\eta\left(x_{\lambda}, a\right)=\lambda \eta(b, a)$, we have

$$
\left\langle F\left(x_{\lambda}\right), \eta\left(x_{\lambda}, a\right)\right\rangle=\left\langle F^{\prime}\left(x_{\lambda}\right)\left(x_{\lambda}-a\right), \eta\left(x_{\lambda}, a\right)\right\rangle=0 .
$$

Hence by (5.15), we have $F^{\prime}\left(x_{\lambda}\right)\left(x_{\lambda}-a\right)=0 . F\left(x_{\lambda}\right)$ must be constant for $\lambda \in[0,1]$. We have $F(a)=F(b)$, that is, $F$ is $\mathrm{IM}_{*}^{+}$on $K$.

Proposition 5.9. Let $K$ be an open convex set, let $F$ be differentiable and IM on $K, \eta$ satisfies Assumptions 5.1, 5.3. For any $x, y \in K$, assume that there hold

$$
\left\langle F^{\prime}(x)(y-x), \eta(y, x)\right\rangle \geq 0, \quad\left\langle F^{\prime}(x)(y-x), \eta(y, x)\right\rangle=0 \Longrightarrow F^{\prime}(x)(y-x)=0 .
$$

Then $F$ is IM+ on K. Furthermore, if $F$ is affine, the condition is necessary as well.

Proof. Assume distinct $a, b \in K$, such that $\langle F(a), \eta(b, a)\rangle=\langle F(b), \eta(b, a)\rangle$. Set $x_{\lambda}=a+$ $\lambda(b-a), \lambda \in[0,1]$. From assumption of $\eta$, we have $\eta\left(x_{\lambda}, a\right)=\lambda \eta(b, a)$ and $\eta\left(x_{\lambda}, b\right)=$ $-(1-\lambda) \eta(b, a)$. The invex monotonicity of $F$ implies that for all $\lambda \in[0,1]$,

$$
\begin{gathered}
0 \leq\left\langle F\left(x_{\lambda}\right)-F(a), \eta\left(x_{\lambda}, a\right)\right\rangle=\lambda\left\langle F\left(x_{\lambda}\right)-F(a), \eta(b, a)\right\rangle, \\
0 \leq\left\langle F\left(x_{\lambda}\right)-F(b), \eta\left(x_{\lambda}, b\right)\right\rangle=-(1-\lambda)\left\langle F\left(x_{\lambda}\right)-F(a), \eta(b, a)\right\rangle,
\end{gathered}
$$

that is, $\left\langle F\left(x_{\lambda}\right), \eta(b, a)\right\rangle=\langle F(a), \eta(b, a)\rangle$. From which we have

$$
\left\langle F^{\prime}\left(x_{\lambda}\right)(b-a), \eta(b, a)\right\rangle=\left\langle F^{\prime}\left(x_{\lambda}\right)\left(x_{\lambda}-a\right), \eta\left(x_{\lambda}, a\right)\right\rangle=0
$$

Considering (5.18), we have $F^{\prime}\left(x_{\lambda}\right)\left(x_{\lambda}-a\right)=\lambda F^{\prime}\left(x_{\lambda}\right)(b-a)=0$. Hence, $F\left(x_{\lambda}\right)$ must be constant for $\lambda \in[0,1]$. We conclude that $F(a)=F(b)$, that is, $F$ is $\mathrm{IM}^{+}$on $K$.

\section{Application to variational-like inequality problem}

In this section we demonstrate the usefulness of the new concepts of generalized invex monotonicity for the study of VLIP, both from the theoretical and computational points of view.

Consider the variational-like inequality $\operatorname{VI}(F, \eta, C)$ characterized by continuous mapping $F$ and function $\eta(x, y)$, that is, we look for a point $x^{*}$ in $C$ that satisfies the variational inequality:

$$
\left\langle F\left(x^{*}\right), \eta\left(x, x^{*}\right)\right\rangle \geq 0 \quad \forall x \in C
$$

where $C$ is convex, compact subset of $\mathscr{R}^{n}$. In the following, we assume that the solution set $\operatorname{Sol}(F, \eta, C)$ of $\operatorname{VI}(F, \eta, C)$ is nonempty.

Lemma 6.1. Let $F$ be PIM on $C$ and $x^{*} \in \operatorname{sol}(F, \eta, C), \eta$ is skew, that is, $\eta(x, y)+\eta(y, x)=0$. Then every solution $\bar{x}$ of $\operatorname{VI}(F, \eta, C)$ lies on the hypersurface $\Gamma^{*}=\left\{y:\left\langle F\left(x^{*}\right), \eta\left(y, x^{*}\right)\right\rangle=\right.$ $0\}$. 
Proof. Let $\bar{x} \in \operatorname{sol}(F, \eta, C)$. By definition of $\operatorname{sol}(F, \eta, C)$ we have

$$
\begin{gathered}
\left\langle F\left(x^{*}\right), \eta\left(\bar{x}, x^{*}\right)\right\rangle \geq 0, \\
\left\langle F(\bar{x}), \eta\left(x^{*}, \bar{x}\right)\right\rangle \geq 0 .
\end{gathered}
$$

From the pseudoinvex monotonicity of $F$ there follows:

$$
\begin{aligned}
\left\langle F(\bar{x}), \eta\left(\bar{x}, x^{*}\right)\right\rangle & \geq 0, \\
\left\langle F\left(x^{*}\right), \eta\left(x^{*}, \bar{x}\right)\right\rangle & \geq 0 .
\end{aligned}
$$

Since $\eta$ is skew, we have $\left\langle F\left(x^{*}\right), \eta\left(\bar{x}, x^{*}\right)\right\rangle=0$.

Proposition 6.2. Let $F$ be a $P^{+} M_{*}^{+}$mapping from $C$ into $\mathscr{R}^{n}$ and $\eta$ is skew. Then the mapping $F$ is constant over the solution set $\operatorname{Sol}(F, \eta, C)$. If $F$ is $P I M_{*}$, then for any $x, y \in$ $\operatorname{Sol}(F, \eta, C)$, there exists $k>0$ such that $F(y)=k F(x)$.

Proof. Let $x$ and $y$ be solutions of $\operatorname{VI}(F, \eta, C)$. By Lemma 6.1, we have that $\langle F(y), \eta(y$, $x)\rangle=0$ and $\langle F(x), \eta(y, x)\rangle=0$. If $F$ is pseudoinvex monotone ${ }_{*}^{+}$, we conclude that $F(y)=$ $F(x)$. If $F$ is pseudoinvex monotone ${ }_{*}$, we conclude that $F(y)=k F(x)$.

Corollary 6.3. Let $F$ be PIM* on $C$ and $\eta$ is skew. If $\bar{x} \in C$ is not a solution of $V I(F, \eta, C)$, then its solution set $\operatorname{Sol}(F, \eta, C)$ lies entirely within the open set $\{x:\langle F(\bar{x}), \eta(x, \bar{x})\rangle<0\}$.

Proposition 6.4. Let $C$ be compact and let F be PIM* and continuous on $C$. $\eta(x, y)$ is skew, continuous in the first argument and satisfies that $\eta(x, y)+\eta(y, z)=\eta(x, z)$. Let $\left\{x_{k}\right\}$ be a sequence of $C$ and $F\left(x_{k}\right)$ a sequence in $\mathscr{R}^{n}$ such that $\lim _{k \rightarrow \infty}\left\langle F\left(x_{k}\right), \eta\left(x_{k}, x^{*}\right)\right\rangle=0$ for some solution $x^{*}$ of $\operatorname{VI}(F, \eta, C)$. Then any limit point $\bar{x}$ of $\left\{x_{k}\right\}$ is a solution of $\operatorname{VI}(F, \eta, C)$.

Proof. Let $\left\{x_{k^{\prime}}\right\}$ be a convergent subsequence of $\left\{x_{k}\right\}$ and $\bar{x}$ its limit point. Since $F$ is bounded, there exists a subsequence $\left\{x_{k^{\prime \prime}}\right\} \subset\left\{x_{k^{\prime}}\right\}$ such that $F\left(x_{k^{\prime \prime}}\right) \rightarrow F(\bar{x})$ and $\langle F(\bar{x})$, $\left.\eta\left(\bar{x}, x^{*}\right)\right\rangle=0$. From the pseudoinvex monotonicity of $F$ we get $\left\langle-F\left(x^{*}\right), \eta\left(\bar{x}, x^{*}\right)\right\rangle \geq 0$. But $x^{*}$ is a solution of $\operatorname{VI}(F, \eta, C)$, thus $\left\langle F\left(x^{*}\right), \eta\left(\bar{x}, x^{*}\right)\right\rangle=0$. Since $F$ is pseudoinvex monotone $_{*}, F(\bar{x})=k F\left(x^{*}\right)$, for some positive number $k$. Finally, for any $x \in C$, we have

$$
\langle F(\bar{x}), \eta(x, \bar{x})\rangle=k\left\langle F\left(x^{*}\right), \eta\left(x, x^{*}\right)\right\rangle+k\left\langle F\left(x^{*}\right), \eta\left(x^{*}, \bar{x}\right)\right\rangle \geq 0 .
$$

This implies that $\bar{x}$ is a solution of $\operatorname{VI}(F, \eta, C)$.

\section{Conclusion}

In this paper, we introduced new forms of generalized invex monotonicity such as (pseudo) invex monotone plus, (pseudo) invex cocoercive, which generalized (pseudo) monotone plus in [2] and (pseudo) cocoercive in [14]. Their relationships, which can be described as shown in Figure 7.1, are discussed by examples and counterexamples. Their differential property is discussed. These new forms allow us to analyze and solve VLIP. 


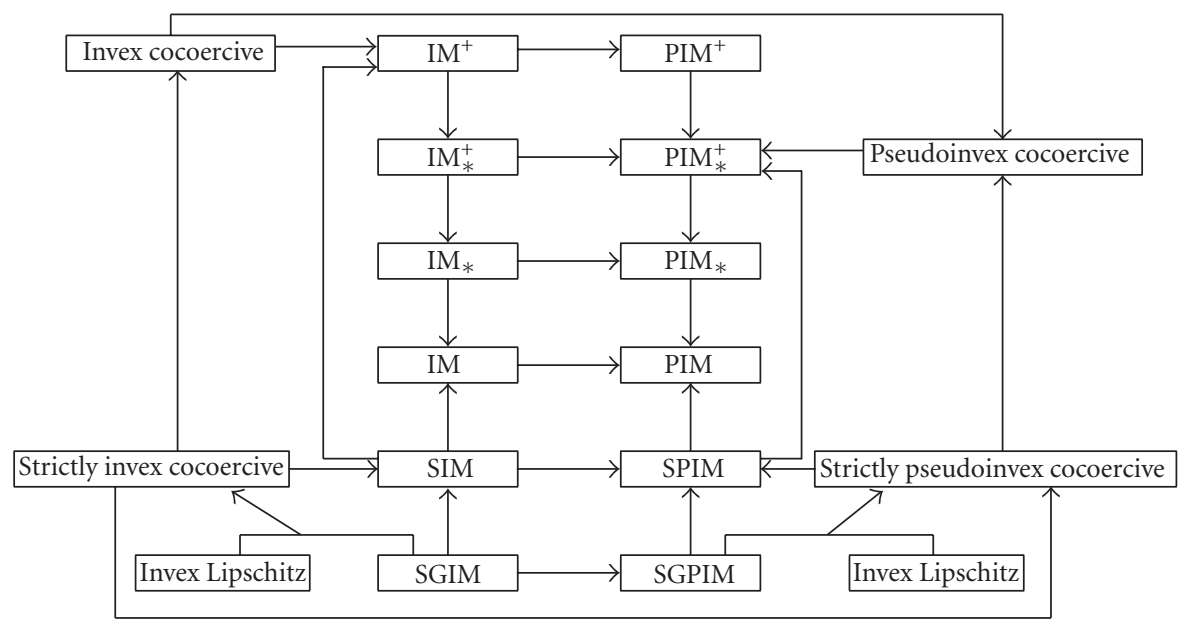

Figure 7.1. Relationships between generalized invex mappings.

\section{Acknowledgments}

This work was partially supported by NSFC 70432001. The authors are indebted to two anonymous referees for their constructive comments and suggestions.

\section{References}

[1] C. Baiocchi and A. Capelo, Variational and Quasivariational Inequalities. Applications to Free Boundary Problems, John Wiley \& Sons, New York, 1984.

[2] J.-P. Crouzeix, P. Marcotte, and D. L. Zhu, Conditions ensuring the applicability of cutting-plane methods for solving variational inequalities, Mathematical Programming. Series A 88 (2000), no. 3, 521-539.

[3] Y. P. Fang and N. J. Huang, Variational-like inequalities with generalized monotone mappings in Banach spaces, Journal of Optimization Theory and Applications 118 (2003), no. 2, 327-338.

[4] P. T. Harker and J.-S. Pang, Finite-dimensional variational inequality and nonlinear complementarity problems: a survey of theory, algorithms and applications, Mathematical Programming. Series B 48 (1990), no. 2, 161-220.

[5] S. Karamardian and S. Schaible, Seven kinds of monotone maps, Journal of Optimization Theory and Applications 66 (1990), no. 1, 37-46.

[6] H. Z. Luo and Z. K. Xu, On characterizations of prequasi-invex functions, Journal of Optimization Theory and Applications 120 (2004), no. 2, 429-439.

[7] S. R. Mohan and S. K. Neogy, On invex sets and preinvex functions, Journal of Mathematical Analysis and Applications 189 (1995), no. 3, 901-908.

[8] R. Osuna-Gómez, A. Rufián-Lizana, and P. Ruíz-Canales, Invex functions and generalized convexity in multiobjective programming, Journal of Optimization Theory and Applications 98 (1998), no. 3, 651-661.

[9] J. Parida, M. Sahoo, and A. Kumar, A variational-like inequality problem, Bulletin of the Australian Mathematical Society 39 (1989), no. 2, 225-231.

[10] G. Ruiz-Garzón, R. Osuna-Gómez, and A. Rufián-Lizana, Generalized invex monotonicity, European Journal of Operational Research 144 (2003), no. 3, 501-512. 
[11] X. Q. Yang, On the gap functions of prevariational inequalities, Journal of Optimization Theory and Applications 116 (2003), no. 2, 437-452.

[12] X. M. Yang, X. Q. Yang, and K. L. Teo, Characterizations and applications of prequasi-invex functions, Journal of Optimization Theory and Applications 110 (2001), no. 3, 645-668.

[13] _ Generalized invexity and generalized invariant monotonicity, Journal of Optimization Theory and Applications 117 (2003), no. 3, 607-625.

[14] D. L. Zhu and P. Marcotte, New classes of generalized monotonicity, Journal of Optimization Theory and Applications 87 (1995), no. 2, 457-471.

[15] Co-coercivity and its role in the convergence of iterative schemes for solving variational inequalities, SIAM Journal on Optimization 6 (1996), no. 3, 714-726.

B. Xu: School of Management, Fudan University, Shanghai 200433, China

Current address: Department of Management Science and Engineering,

Nanchang University, Jiangxi 330047, China

E-mail address: xu_bing99@sina.com

D. L. Zhu: School of Management, Fudan University, Shanghai 200433, China

E-mail address: d.l.zhu@fudan.edu.cn 